\title{
一种硫醚氧化为砜的绿色合成方法
}

\author{
方向*, $a$ 王 旺 ${ }^{a}$ 杨雪艳 ${ }^{a}$ 吴范宏*,b \\ ( ${ }^{a}$ 华东理工大学化学与分子工程学院 上海 200237) \\ $\left({ }^{b}\right.$ 上海应用技术大学 上海绿色氟代制药工程技术研究中心 上海 201418)
}

\begin{abstract}
摘要 硫梄在 Selectfluor ${ }^{\mathrm{TM}}$ 的作用下，以水作为氧源得到相应砜类化合物，最高收率可达 $99 \%$. 在重氧水标记下，得到 ${ }^{18} \mathrm{O}$ 标记的甲基苯基砜产物. 本方法绿色环保, 条件温和, 并可放大至克量级规模.

关键词 硫醚; Selecetfluor ${ }^{\mathrm{TM}}$; 水; 砜类化合物
\end{abstract}

\section{A Green Method for the Synthesis of Sulfones from Thioethers}

\author{
Fang, Xiang ${ }^{*, a} \quad$ Wang, Wang ${ }^{a} \quad$ Yang, Xueyan $^{a} \quad$ Wu, Fanhong ${ }^{*, b}$ \\ ( ${ }^{a}$ School of Chemistry and Molecular Engineering, East China University of Science and Technology, Shanghai 200237) \\ ( ${ }^{b}$ Shanghai Engineering Research Center of Green Fluoropharmaceutical Technology, Shanghai Institute of \\ Technology, Shanghai 201418)
}

\begin{abstract}
With water as oxygen source, a method for the synthesis of sulfones from thioethers under the action of Selecetfluor ${ }^{\mathrm{TM}}$ was designed. The yield can reach $99 \%$. The ${ }^{18} \mathrm{O}$-labeled methylphenylsulfone was prepared with $\mathrm{H}_{2}{ }^{18} \mathrm{O}$. The conditions of this green method are mild and can reach the grams scale.

Keywords thioethers; Selecetfluor ${ }^{\mathrm{TM}}$; water; sulfone compound
\end{abstract}

砜基作为有机官能团，在农药 ${ }^{[1]}$ 、天然产物 ${ }^{[2]}$ 和医 药 ${ }^{[3]}$ 领域, 具有重要的应用价值. 砜是燃料脱硫的重要 中间体 ${ }^{[4]}$, 硫氧化作用也是原油催化氧化脱硫脱除硫基 杂质的基础. 此外, 含砜基的化合物在苂光光致变色领 域也有重要的研究意义 ${ }^{[5]}$.

目前将硫醚氧化为相应砜类化合物的方法已非常 成熟, 如实验室或工业生产中最常见的双氧水氧化 ${ }^{[6]}$. 除双氧水氧化以外, 还有壬二酸 ${ }^{[7]}$ 、过氧化叔丁醇 $(\mathrm{TBHP})^{[8]}$ 、过氧化腿 $(\mathrm{UHP})^{[9]}$ 、间氯过氧苯甲酸 $(m-\mathrm{CPBA})^{[10]}$ 等氧化方法以及金属辅助催化氧化( 诸如 $\mathrm{Sb}^{[11]}, \mathrm{W}^{[12]}, \mathrm{Ru}^{[13]}, \mathrm{Re}^{[14]}, \mathrm{V}^{[15]}, \mathrm{Mo}^{[16]}$ 等). 然而, 这些方 法在安全性、毒性、反应的可操作性和重金属的去除方 面仍存在一些缺陷, 因此, 更方便和环境友好的氧化方 法仍是必不可少的.

亲电氟化试剂 Selectfluor ${ }^{\mathrm{TM}}$ 是一种白色的固体, 易 储存, 且可通过商业途径获得. Selectfluor ${ }^{\mathrm{TM}}$ 在有机合成 中有着广泛的利用 ${ }^{[17]}$. Vincent 等 ${ }^{[18]}$ 发现在室温下, 各种
硫苷在 Selectfluor ${ }^{\mathrm{TM}}$ 作用下很容易转化为其亚砜基衍生 物. Masayuki Kirihara 等 ${ }^{[19]}$ 用 Selectfluor ${ }^{\mathrm{TM}}$ 作用将二硫化 物氧化为硫代磺酸酯和苯基磺酰氟. 我们设想硫梄类化 合物是否也能在 Selecetfluor ${ }^{\mathrm{TM}}$ 的作用下, 在温和条件 下，得到相应的砜类化合物.

\section{1 结果与讨论}

以甲基苯基硫醚(1a)作为反应底物, 使用过量的 Selectfluor $^{\mathrm{TM}}$ (2), 对反应条件进行优化. 结果发现, 硫 醚的氧化反应在常用的有机溶剂中并不发生(表 1, Entries 1 9), 而当用水作为溶剂, 惊喜地以 $99 \%$ 的产率得 到氧化产物甲基苯基砜(3a)(表 1, Entry 10). Selectfluor ${ }^{\mathrm{TM}}$ 用量降低后，甲基苯基砜的产率会有所下降(表 1, Entry 11 ), 进一步将 Selectfluor ${ }^{\mathrm{TM}}$ 用量降为 $\mathbf{1 a}$ 的 1.5 倍时, 甲 基苯基砜的产率锐减为 46\%(表 1, Entry 12).

由于硫醚只在水相中才氧化为砜, 而 Selectfluor ${ }^{\mathrm{TM}}$ 本身不含氧, 因此我们推测氧化产物砜基中的氧可能来

* Corresponding authors. E-mail: fangxiang@ecust.edu.cn; fangxiang@ecust.edu.cn Received July 9, 2020; revised July 30, 2020; published online August 26, 2020. Project supported by the National Natural Science Foundation of China (No. 21672151). 国家自然科学基金(No. 21672151)资助项目. 
表 1 反应条件的优化 ${ }^{a}$

Table 1 Optimization of Reaction Conditions

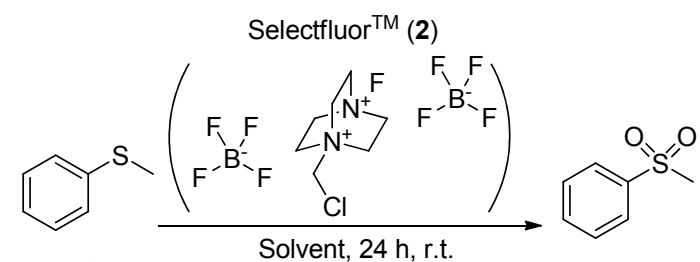

$1 \mathrm{a}$

Solvent, 24 h, r.t.

$3 a$

\begin{tabular}{clc}
\hline Entry & \multicolumn{1}{c}{ Solvent } & Yield $^{b} \%$ \\
\hline 1 & $\mathrm{MeOH}^{\circ}$ & - \\
2 & $\mathrm{CH}_{2} \mathrm{Cl}_{2}$ & - \\
3 & $\mathrm{CHCl}_{3}$ & - \\
4 & $\mathrm{CH}_{3} \mathrm{COOEt}$ & - \\
5 & $\mathrm{CH}_{3} \mathrm{CN}$ & - \\
6 & $\mathrm{EtOH}$ & - \\
7 & $\mathrm{THF}$ & - \\
8 & $\mathrm{DMF}$ & - \\
9 & $\mathrm{PE}$ & 99 \\
10 & $\mathrm{H}_{2} \mathrm{O}$ & $90^{c}$ \\
11 & $\mathrm{H}_{2} \mathrm{O}$ & $46^{d}$ \\
12 & $\mathrm{H}_{2} \mathrm{O}$ & 2 \\
\hline
\end{tabular}

${ }^{a}$ Reaction conditions: 1a $(1 \mathrm{mmol}), \mathbf{2}(2.2 \mathrm{mmol})$, solvent $2 \mathrm{~mL}, 24$ h, r.t.; ${ }^{b}$ Isolated yield; ${ }^{c}$ Reaction conditions: 1a $(1 \mathrm{mmol}), 2(2 \mathrm{mmol})$, solvent $2 \mathrm{~mL}$, 24 h, r.t.; ${ }^{d}$ Reaction conditions: 1a $(1 \mathrm{mmol}), 2$ (1.5 mmol), solvent $2 \mathrm{~mL}, 24$ h, r.t.
源于水. 胡金波等 ${ }^{[20]}$ 曾经报道了在重氧水标记下，偕二 氟烯烃和 Selectfluor ${ }^{\mathrm{TM}}$ 反应得到 ${ }^{18} \mathrm{O}$ 标记的 $\alpha$-三氟甲基 醇. 为验证我们的推测结果, 采用同样的方法, 将反应 置于 10 equiv.的重氧水 $(10 \mathrm{mmol})$ 中进行, 结果以 $95 \%$ 的 收率得到含有 ${ }^{18} \mathrm{O}$ 标记的甲基苯基砜产物, 确定砜基中 的氧确实来源于水(Scheme 1).

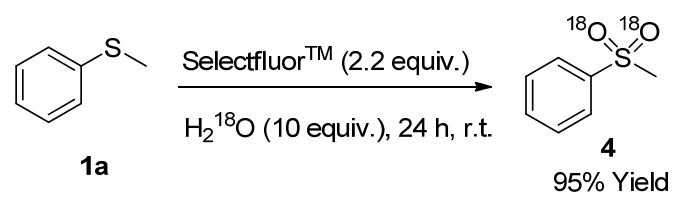

图式 1 氧源实验

Scheme 1 Oxygen source experiment

确定了砜基中氧的来源后, 进一步对反应底物进行 了拓展，考察电子效应和位阻效应对反应的影响. 无论 苯环上是给电子基团还是吸电子基团，反应的收率都超 过 $90 \%$. 值得注意的是，当底物围绕硫原子的位阻增大 时, 反应需要在更高的温度下进行, 相应的产率也有所 下降(表 2, Entries 7,8).

为了进一步研究反应机理, 首先用 1 equiv. 甲基苯 基硫醚(1a)与 1.1 equiv. Selectfluor ${ }^{\mathrm{TM}}(1.1 \mathrm{mmol})$ 反应, 以

表 2 反应底物普适性研究 ${ }^{a}$

Table 2 Evaluation of Substrate Scope

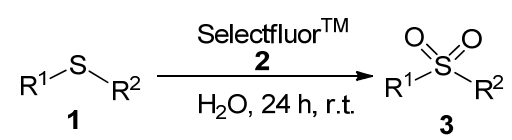

Entry Substrate




\begin{tabular}{|c|c|c|c|c|}
\hline Entry & Substrate & Product & Yield $^{b} / \%$ & m.p. $/{ }^{\circ} \mathrm{C}$ \\
\hline 5 & $1 e$ & $3 e$ & 99 & $118.5 \sim 119.5\left(119 \sim 120^{[21]}\right)$ \\
\hline 6 & & $3 \mathrm{f}$ & 95 & $107 \sim 108.2\left(105 \sim 106^{[22]}\right)$ \\
\hline 7 & & & $89^{c}$ & $124 \sim 125\left(121 \sim 126^{[9]}\right)$ \\
\hline 8 & 1h & 3h & $56^{c}$ & $235 \sim 236.6\left(234 \sim 237^{[9]}\right)$ \\
\hline 9 & $1 \mathbf{i}$ & $3 \mathbf{i}$ & $97^{d}$ & $76.8 \sim 77.5\left(76^{[23]}\right)$ \\
\hline 10 & 1j & $3 \mathbf{j}$ & $95^{d}$ & $139.2 \sim 140.8\left(139 \sim 141^{[24]}\right)$ \\
\hline 11 & $1 \mathrm{k}$ & 3k & $94^{d}$ & $140.1 \sim 141.6\left(140.0 \sim 142.1^{[25]}\right)$ \\
\hline
\end{tabular}

${ }^{a}$ Reaction conditions: $1(10 \mathrm{mmol}), 2(25 \mathrm{mmol}), \mathrm{H}_{2} \mathrm{O}(5 \mathrm{~mL}), 24 \mathrm{~h}$, r.t.; ${ }^{b}$ Isolated yield; ${ }^{c}$ Reaction conditions: $1(10 \mathrm{mmol}), 2(25 \mathrm{mmol}), \mathrm{H}_{2} \mathrm{O}(5 \mathrm{~mL}), 24 \mathrm{~h}, 60{ }^{\circ} \mathrm{C}$; ${ }^{d}$ Reaction conditions: 1 ( $\left.2 \mathrm{mmol}\right), 2(5 \mathrm{mmol}), \mathrm{H}_{2} \mathrm{O}(2 \mathrm{~mL}), 24$ h, r.t.

$84 \%$ 的产率获得了甲基苯基亚砜(5) (Scheme 2, a). 随后 又用 1 equiv. 甲基苯基亚砜(5)与 1.1 equiv. Selectfluor ${ }^{\mathrm{TM}}$ (1.1 mmol)反应, 又顺利地得到了甲基苯基砜(3a), 产率 为 $86 \%$ (Scheme 2, b). 接着, 在反应中加入 5 equiv. 四 甲基哌啶氧化物(TEMPO) (5 mmol)进行自由基捕捉实 验，但仍以 $82 \%$ 的收率得到甲基苯基砜 3a (Scheme 2, c), 说明此反应历程并不是自由基反应.

为此, 我们给出可能的反应途径(Scheme 3). 首先, 甲基苯基硫醚(1a)经 Selectfluor ${ }^{\mathrm{TM}}$ 氟化, 水中的氧原子 进攻硫原子形成中间态 $\mathbf{A}$. 随后 $\mathbf{A}$ 经过脱除 $\mathrm{HF}$ 和 $\mathrm{H}^{+}$ 得到甲基苯基亚砜(5). 甲基苯基亚砜(5)经过类似的机 理进一步氧化最终得到甲基苯基砜(3a). (a)<smiles>CS(=O)c1cccc(N=C2OC3CCCCC23)c1</smiles>

(b)<smiles>CS(=O)(=O)c1ccc(I)cc1</smiles>

(c)

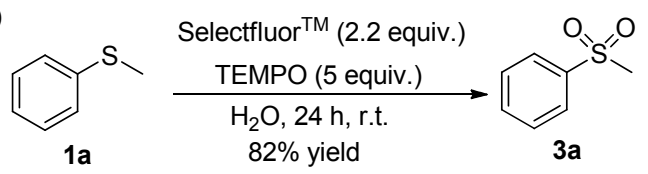

图式 2 控制实验

Scheme 2 Control experiments 


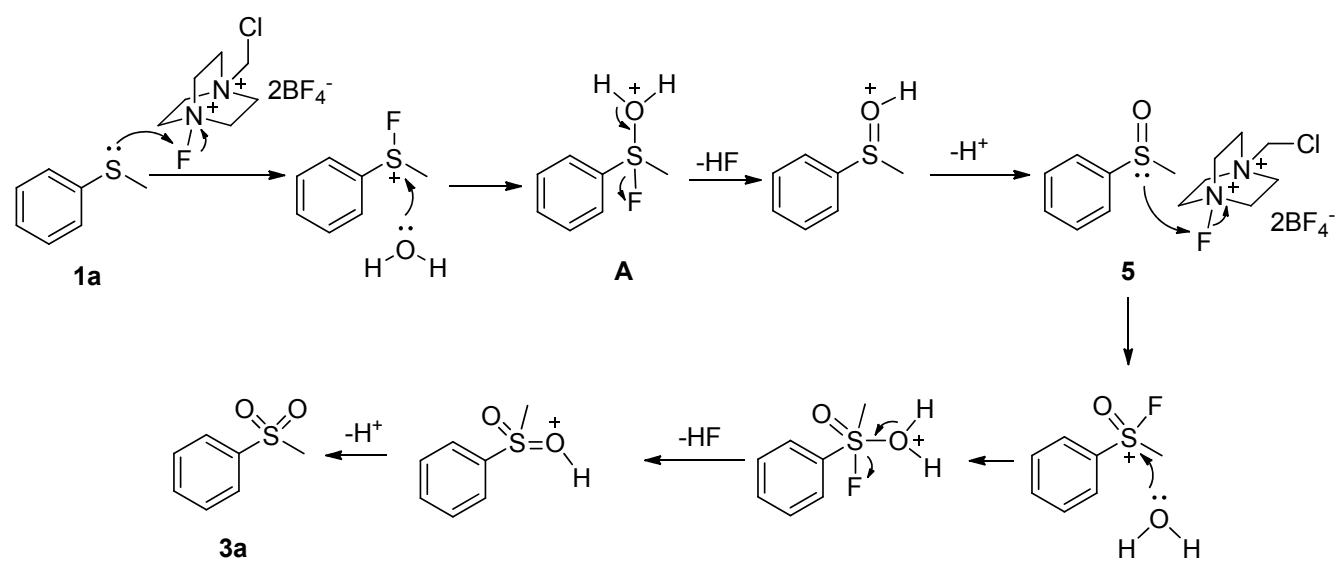

图式 3 可能的反应途径

Scheme 3 Proposed reaction pathway

\section{2 结论}

综上所述, 设计了一种在 Selectfluor ${ }^{\mathrm{TM}}$ 作用下, 以 水作为氧源, 硫醚氧化为砜的绿色合成方法, 并获得良 好收率. 此合成方法以水为溶剂和氧源, 反应条件温和, 避免了较危险的双氧水的使用, 反应规模可放大至克量 级. 我们将对此合成方法产量的进一步放大进行更深入 的研究.

\section{3 实验部分}

\section{1 仪器与试剂}

熔点由 SGW X-4A 显微熔点仪测定. ${ }^{1} \mathrm{H}$ NMR (400 $\mathrm{MHz}$ )和 ${ }^{13} \mathrm{C}$ NMR (100 MHz) 数据皆是用 Bruker Avance 400 型核磁共振仪测试所得, 所用溶剂均为 $\mathrm{CDCl}_{3}$. 高 分辨质谱数据由 Waters-GCT 质谱仪测定. 此外, 若无 特殊说明, 所用的试剂皆为商业购买获得.

\section{2 实验方法}

\subsection{1 由硫醚化合物 $\mathbf{1}$ 制备砜类化合物 $\mathbf{3}$}

将硫醚化合物 $\mathbf{1}(10 \mathrm{mmol}) 、 5 \mathrm{~mL} \mathrm{H} \mathrm{H}_{2} \mathrm{O}$ 、Selectfluor ${ }^{\mathrm{TM}}$ $2(25 \mathrm{mmol})$ 依次加入到 $25 \mathrm{~mL}$ 的圆底烧瓶中, 在室温下 $\left(1 \mathrm{~g}\right.$ 和 $1 \mathrm{~h}$ 的反应温度为 $\left.60{ }^{\circ} \mathrm{C}\right)$ 摚拌 $24 \mathrm{~h}$. 由于反应是两 相反应, 烧瓶中存在大量固体, 需要进行充分搅拌. 反 应结束后, 加入饱和碳酸氢钠溶液, 调节 $\mathrm{pH}$ 至弱碱性, 用乙酸乙酯萃取. 萃取后取有机层, 加入适量的无水硫 酸钠干燥 $6 \mathrm{~h}$. 干燥后, 旋蒸浓缩, 进行柱层析分离(展 开剂: 石油醚/乙酸乙酯, $V: V=4: 1$ ), 得到克量级的 相应的砜类化合物 $\mathbf{3}$.

甲基苯基砜(3a): $1.532 \mathrm{~g}$ 白色固体，产率 98\%. m.p. $84.5 \sim 85.6{ }^{\circ} \mathrm{C} ;{ }^{1} \mathrm{H}$ NMR $\left(\mathrm{CDCl}_{3}, 400 \mathrm{MHz}\right) \delta$ : $7.95(\mathrm{~d}$, $J=8.1 \mathrm{~Hz}, 2 \mathrm{H}), 7.67(\mathrm{t}, J=7.4 \mathrm{~Hz}, 1 \mathrm{H}), 7.58(\mathrm{t}, J=7.7$ $\mathrm{Hz}, 2 \mathrm{H}), 3.07$ (s, 3H); ${ }^{13} \mathrm{C} \mathrm{NMR}\left(\mathrm{CDCl}_{3}, 101 \mathrm{MHz}\right) \delta$ :
140.51, 133.74, 129.38, 127.25, 44.41; HRMS (EI) calcd for $\mathrm{C}_{7} \mathrm{H}_{8} \mathrm{O}_{2} \mathrm{~S} 156.0245$, found 156.0246 .

对甲基苯甲砜(3b)：1.680 $\mathrm{g}$ 白色固体，产率 99\%. m.p. 85.5 86.8 ${ }^{\circ} \mathrm{C} ;{ }^{1} \mathrm{H}$ NMR $\left(\mathrm{CDCl}_{3}, 400 \mathrm{MHz}\right) \delta: 7.82$ (d, $J=7.2 \mathrm{~Hz}, 2 \mathrm{H}), 7.36$ (d, $J=7.4 \mathrm{~Hz}, 2 \mathrm{H}), 3.04$ (s, 3H), 2.45 (s, 3H); ${ }^{13} \mathrm{C}$ NMR $\left(\mathrm{CDCl}_{3}, 101 \mathrm{MHz}\right) \delta: 144.68$, 137.65, 129.96, 127.32, 44.58, 21.61; HRMS (EI) calcd for $\mathrm{C}_{8} \mathrm{H}_{10} \mathrm{O}_{2} \mathrm{~S} 170.0402$, found 170.0403 .

4-氯苯甲砜(3c): $1.860 \mathrm{~g}$ 白色固体, 产率 $98 \%$. m.p. 95 96 ${ }^{\circ} \mathrm{C} ;{ }^{1} \mathrm{H}$ NMR $\left(\mathrm{CDCl}_{3}, 400 \mathrm{MHz}\right) \delta: 7.90(\mathrm{~d}, J=8.6$ $\mathrm{Hz}, 2 \mathrm{H}), 7.56$ (d, $J=8.6 \mathrm{~Hz}, 2 \mathrm{H}), 3.07$ (s, 3H); ${ }^{13} \mathrm{C} \mathrm{NMR}$ $\left(\mathrm{CDCl}_{3}, 101 \mathrm{MHz}\right) \delta: 140.35,139.00,129.70,128.92$, 44.48; HRMS (EI) calcd for $\mathrm{C}_{7} \mathrm{H}_{7} \mathrm{ClO}_{2} \mathrm{~S} 189.9855$, found 189.9852 .

4-溴苯甲砜(3d): $2.295 \mathrm{~g}$ 白色固体, 产率 98\%. m.p. $101.4 \sim 102.3{ }^{\circ} \mathrm{C} ;{ }^{1} \mathrm{H}$ NMR $\left(\mathrm{CDCl}_{3}, 400 \mathrm{MHz}\right) \delta: 7.78(\mathrm{dd}$, $J=8.5,1.2 \mathrm{~Hz}, 2 \mathrm{H}), 7.68(\mathrm{dd}, J=8.5,1.7 \mathrm{~Hz}, 2 \mathrm{H}), 3.04(\mathrm{~s}$, $3 \mathrm{H}) ;{ }^{13} \mathrm{C}$ NMR $\left(\mathrm{CDCl}_{3}, 101 \mathrm{MHz}\right) \delta: 139.52,132.69$, 128.98, 128.93, 44.45; HRMS (EI) calcd for $\mathrm{C}_{7} \mathrm{H}_{7} \mathrm{BrO}_{2} \mathrm{~S}$ 233.9350 , found 233.9352 .

4-甲氧基苯甲砜(3e)：1.850 g 白色固体，产率 99\%. m.p. $118.5 \sim 119.5{ }^{\circ} \mathrm{C} ;{ }^{1} \mathrm{H}$ NMR $\left(\mathrm{CDCl}_{3}, 400 \mathrm{MHz}\right) \delta$ : 7.87 (dd, $J=8.7,1.6 \mathrm{~Hz}, 2 \mathrm{H}), 7.03(\mathrm{~d}, J=8.8 \mathrm{~Hz}, 2 \mathrm{H})$, 3.89 (s, 3H), 3.04 (s, 3H); ${ }^{13} \mathrm{C}$ NMR $\left(\mathrm{CDCl}_{3}, 101 \mathrm{MHz}\right) \delta$ : 163.70, 132.23, 129.53, 114.51, 55.73, 44.85; HRMS (EI) calcd for $\mathrm{C}_{8} \mathrm{H}_{10} \mathrm{O}_{3} \mathrm{~S} 186.0351$, found 186.0349 .

2-澳苯甲砜(3f): $2.225 \mathrm{~g}$ 白色固体, 产率 $95 \%$. m.p. $107 \sim 108.2{ }^{\circ} \mathrm{C} ;{ }^{1} \mathrm{H}$ NMR $\left(\mathrm{CDCl}_{3}, 400 \mathrm{MHz}\right) \delta: 8.20(\mathrm{dd}$, $J=7.7,1.9 \mathrm{~Hz}, 1 \mathrm{H}), 7.78(\mathrm{dd}, J=7.7,1.3 \mathrm{~Hz}, 1 \mathrm{H}), 7.56 \sim$ 7.47 (m, 2H), 3.30 (s, 3H); ${ }^{13} \mathrm{C} \mathrm{NMR}\left(\mathrm{CDCl}_{3}, 101 \mathrm{MHz}\right) \delta$ : 139.56, 135.46, 134.88, 131.08, 128.19, 120.58, 42.41; HRMS (EI) calcd for $\mathrm{C}_{7} \mathrm{H}_{7} \mathrm{BrO}_{2} \mathrm{~S}$ 233.9350, found 
233.9349 .

二苯砜 (3g): $1.935 \mathrm{~g}$ 白色固体, 产率 $89 \%$. m.p. 124 125 ${ }^{\circ} \mathrm{C} ;{ }^{1} \mathrm{H}$ NMR $\left(\mathrm{CDCl}_{3}, 400 \mathrm{MHz}\right) \delta$ : 7.95 (dd, $J=7.1,1.5 \mathrm{~Hz}, 4 \mathrm{H}), 7.58 \sim 7.44(\mathrm{~m}, 6 \mathrm{H}) ;{ }^{13} \mathrm{C} \mathrm{NMR}$ $\left(\mathrm{CDCl}_{3}, 101 \mathrm{MHz}\right) \delta: 141.57,133.25,129.32,127.64$; HRMS (EI) calcd for $\mathrm{C}_{12} \mathrm{H}_{10} \mathrm{O}_{2} \mathrm{~S}$ 218.0402, found 218.0401 .

二苯并噻吩砜(3h): $1.212 \mathrm{~g}$ 白色固体, 产率 $56 \%$. m.p. $235 \sim 236.6{ }^{\circ} \mathrm{C} ;{ }^{1} \mathrm{H}$ NMR $\left(\mathrm{CDCl}_{3}, 400 \mathrm{MHz}\right) \delta$ : $7.81 \sim 7.75(\mathrm{~m}, 4 \mathrm{H}), 7.61(\mathrm{td}, J=7.6,1.1 \mathrm{~Hz}, 2 \mathrm{H}), 7.50$ (td, $J=7.6,0.9 \mathrm{~Hz}, 2 \mathrm{H}) ;{ }^{13} \mathrm{C} \mathrm{NMR}\left(\mathrm{CDCl}_{3}, 101 \mathrm{MHz}\right) \delta$ : $137.62,133.95,131.58,130.39,122.12,121.66$; HRMS (EI) calcd for $\mathrm{C}_{12} \mathrm{H}_{8} \mathrm{O}_{2} \mathrm{~S} 216.0245$, found 216.0244.

4-氟苯甲砜 (3i): $0.336 \mathrm{~g}$ 白色固体, 产率 97\%. m.p. $76.8 \sim 77.5{ }^{\circ} \mathrm{C} ;{ }^{1} \mathrm{H}$ NMR $\left(400 \mathrm{MHz}, \mathrm{CDCl}_{3}\right) \delta: 7.98(\mathrm{dd}$, $J=8.8,5.0 \mathrm{~Hz}, 2 \mathrm{H}), 7.26$ (t, $J=8.5 \mathrm{~Hz}, 2 \mathrm{H}), 3.07$ (s, $3 \mathrm{H}$ ); ${ }^{13} \mathrm{C}$ NMR (101 MHz, $\left.\mathrm{CDCl}_{3}\right) \delta: 165.79(\mathrm{~d}, J=256.2 \mathrm{~Hz})$, 136.68 (d, $J=3.2 \mathrm{~Hz}), 130.31$ (d, $J=9.6 \mathrm{~Hz}), 116.70$ (d, $J=22.7 \mathrm{~Hz}$ ), 44.67; HRMS (EI) calcd for $\mathrm{C}_{7} \mathrm{H}_{7} \mathrm{FO}_{2} \mathrm{~S}$ 174.0151, found 174.0152.

4-硝基苯甲砜 $(\mathbf{3 j}$ ): $0.382 \mathrm{~g}$ 淡黄色固体, 产率 95\%. m.p. $139.2 \sim 140.8{ }^{\circ} \mathrm{C} ;{ }^{1} \mathrm{H}$ NMR $\left(400 \mathrm{MHz}, \mathrm{CDCl}_{3}\right) \delta$ : $8.44(\mathrm{~d}, J=8.9 \mathrm{~Hz}, 2 \mathrm{H}), 8.18(\mathrm{~d}, J=8.9 \mathrm{~Hz}, 2 \mathrm{H}), 3.14$ (s, $3 \mathrm{H}) ;{ }^{13} \mathrm{C}$ NMR $\left(101 \mathrm{MHz}, \mathrm{CDCl}_{3}\right) \delta: 150.87,145.95$, 129.00, 124.66, 44.29; HRMS (EI) calcd for $\mathrm{C}_{7} \mathrm{H}_{7} \mathrm{NO}_{4} \mathrm{~S}$ 201.0096, found 201.0097.

4-氰基苯甲砜(3k): $0.339 \mathrm{~g}$ 白色固体, 产率 $94 \%$. m.p. $140.1 \sim 141.6{ }^{\circ} \mathrm{C} ;{ }^{1} \mathrm{H}$ NMR $\left(400 \mathrm{MHz}, \mathrm{CDCl}_{3}\right) \delta$ : $8.10(\mathrm{~d}, J=8.2 \mathrm{~Hz}, 2 \mathrm{H}), 7.92(\mathrm{~d}, J=8.2 \mathrm{~Hz}, 2 \mathrm{H}), 3.12$ (s, $3 \mathrm{H}) ;{ }^{13} \mathrm{C}$ NMR (101 MHz, $\left.\mathrm{CDCl}_{3}\right) \delta: 144.45,133.25$, 128.21, 117.53, 117.13, 44.20; HRMS (EI) calcd for $\mathrm{C}_{8} \mathrm{H}_{7} \mathrm{~N}-\mathrm{O}_{2} \mathrm{~S} 181.0198$, found 181.0199.

\subsection{2 ${ }^{18} \mathrm{O}$-甲基苯基砜(4)的合成}

将甲基苯基硫醚(1a) (1 mmol)、重氧水(10 mmol)、 Selectfluor $^{\mathrm{TM}}$ (2.2 mmol)依次加入到反应管中, 在室温 下搅拌 $24 \mathrm{~h}$. 反应结束后, 加入饱和碳酸氢钠溶液, 调 节 $\mathrm{pH}$ 至弱碱性, 用乙酸乙酯萃取. 萃取后取有机层, 加 入适量的无水硫酸钠干燥 $6 \mathrm{~h}$. 干燥后, 旋蒸浓缩, 进行 柱层析分离 (展开剂: 石油醚/乙酸乙酯, $V: V=4: 1$ ), 得到 $0.152 \mathrm{~g}$ 白色固体 ${ }^{18} O$-甲基苯基砜(4), $O$-甲基苯基砜 (4): $0.152 \mathrm{~g}$ 白色固体, 产率 95\%. m.p. 85.0 86.2 ${ }^{\circ} \mathrm{C} ;{ }^{1} \mathrm{H}$ NMR (400 MHz, $\left.\mathrm{CDCl}_{3}\right) \delta: 7.96(\mathrm{~d}, J=7.3 \mathrm{~Hz}, 2 \mathrm{H}), 7.67$ (t, $J=7.4 \mathrm{~Hz}, 1 \mathrm{H}), 7.58$ (t, $J=7.6 \mathrm{~Hz}, 2 \mathrm{H}), 3.06$ (s, 3H); ${ }^{13} \mathrm{C}$ NMR $\left(101 \mathrm{MHz}, \mathrm{CDCl}_{3}\right) \delta: 140.56,133.73,129.39$, 127.34, 44.48; HRMS (EI) calcd for $\mathrm{C}_{7} \mathrm{H}_{8}{ }^{18} \mathrm{O}_{2} \mathrm{~S} 160.0330$, found 160.0332 .

3.2.3 由苯甲硫醚(1a)制备甲基苯基亚砜(5)

将甲基苯基硫醚(1a) $\left(1 \mathrm{mmol}\right.$ )、2 $\mathrm{mL} \mathrm{H}_{2} \mathrm{O}$ 、 Selectfluor $^{\mathrm{TM}}(1.1 \mathrm{mmol})$ 依次加入到反应管中, 在室温 下搅拌 $24 \mathrm{~h}$. 反应结束后, 加入饱和碳酸氢钠溶液, 调 节 $\mathrm{pH}$ 至弱碱性，用乙酸乙酯萃取. 萃取后取有机层，加 入适量的无水硫酸钠干燥 $6 \mathrm{~h}$. 干燥后, 旋蒸浓缩, 进行 柱层析分离(展开剂: 石油醚/乙酸乙酯, $V: V=4: 1$ ), 得到 $0.118 \mathrm{~g}$ 无色液体甲基苯基亚砜(5), 产率 $84 \% .{ }^{1} \mathrm{H}$ NMR $\left(\mathrm{CDCl}_{3}, 400 \mathrm{MHz}\right) \delta: 7.66(\mathrm{dd}, J=7.7,1.5 \mathrm{~Hz}, 2 \mathrm{H})$, $7.56 \sim 7.49(\mathrm{~m}, 3 \mathrm{H}), 2.73(\mathrm{~s}, 3 \mathrm{H}) ;{ }^{13} \mathrm{C} \mathrm{NMR}\left(\mathrm{CDCl}_{3}, 101\right.$ MHz) $\delta: 145.63,131.05,129.36,123.48,43.94$; HRMS (EI) calcd for $\mathrm{C}_{7} \mathrm{H}_{8} \mathrm{OS} 140.0296$, found 140.0297 .

3.2.4 由甲基苯基亚砜(5)制备甲基苯基砜(3a)

将甲基苯基亚砜(5) $(1 \mathrm{mmol}) 、 2 \mathrm{~mL} \mathrm{H}_{2} \mathrm{O}$ 、Selectfluor $^{\mathrm{TM}}(1.1 \mathrm{mmol})$ 依次加入到反应管中, 在室温下搅拌 $24 \mathrm{~h}$. 反应结束后, 加入饱和碳酸氢钠溶液, 调节 $\mathrm{pH}$ 至 弱碱性, 用乙酸乙酯萃取. 萃取后取有机层, 加入适量 的无水硫酸钠干燥 $6 \mathrm{~h}$. 干燥后, 旋蒸浓缩, 进行柱层析 分离(展开剂: 石油醚/乙酸乙酯, $V: V=4: 1$ )得到 $0.134 \mathrm{~g}$ 白色固体甲基苯基砜(3a).

辅助材料(Supporting Information) 化合物 $3 \mathrm{a} \sim 3 \mathrm{k}, 4$ 和 5 的 ${ }^{1} \mathrm{H}$ NMR 和 ${ }^{13} \mathrm{C}$ NMR 谱图. 这些材料可以免费从 本刊网站(http://sioc-journal.cn/)上下载.

\section{References}

[1] Devendar, P.; Yang, G. F. Sulfur Chemistry, Springer, Cham, 2019, pp. $35 \sim 78$.

[2] Waldman, A. J.; Ng, T. L.; Wang, P.; Balskus, E. P. Chem. Rev. 2017, 117, 5784 .

[3] Feng, M.; Tang, B.; H. Liang, S. H.; Jiang, X. Curr. Top. Med. Chem. 2016, 16, 1200.

[4] Wang, D.; Qian, E. W.; Amano, H.; Okata, K.; Ishihara, A.; Kabe, T. Appl. Catal., A 2003, 253, 91.

[5] (a) Taguchi, M.; Nakagawa, T.; Nakashima, T.; Kawai, T. J. Mater. Chem. 2011, 21, 17425.

(b) Takagi, Y.; Kunishi, T.; Katayama, T.; Ishibashi, Y.; Miyasaka, H.; Morimoto, M.; Irie, M. Photochem. Photobiol. Sci. 2012, 11, 1661.

[6] Zhang, J.; Mai, Y.; Jiang, T.; Wang, X.; Chen, J.; Zhang, L.; Gao, M.; Li, Y. CN 108586297, 2018

[7] Gayakwad, E. M.; Patil, V. V.; Shankarling, G. S. New J. Chem. 2016, 40, 223.

[8] Zaburdaeva, E.; Dodonov, V.; Stepovik, L.; Kapuskina, I. Phosphorus, Sulfur Silicon Relat. Elem. 2005, 180, 1521.

[9] Lutz, M.; Wenzler, M.; Likhotvorik, I. Synthesis 2018, 50, 2231.

[10] (a) Zheng, S.; Huang, W.; Gao, N.; Cui, R.; Zhang, M.; Zhao, X. Chem. Commun. 2011, 47, 6969.

(b) Zhang, M.; Zhao, M.; Zheng, P.; Zhang, H.; Zhao, X. J. Fluorine Chem. 2016, 189, 13.

(c) Gao, N.; Zheng, S.; Yang, W.; Zhao, X. Org. Lett. 2011, 13, 1514.

(d) Gao, N.; Guo, X.; Zheng, S.-C.; Yang, W.-K.; Zhao, X.-M. Tet- 
rahedron 2012, 68, 9413.

[11] Lu, J.; Wang, Y.; Ma, X.; Niu, Y.; Singh, V.; Ma, P.; Zhang, C.; Niu, J.; Wang, J. Dalton Trans. 2018, 47, 8070.

[12] Koo, D. H.; Kim, M.; Chang, S. Org. Lett. 2005, 7, 5015.

[13] Ali, M. H.; Olesen, B.; Ranu, B.; Clippard, L.; Heath, J.; Meyer, G.; Williams, T. Synthesis 2016, 48, 429.

[14] Yamazaki, S. Bull. Chem. Soc. Jpn. 1996, 69, 2955.

[15] Mohammadpoor-Baltork, I.; Hill, M.; Caggiano, L.; Jackson, R. F. W. Synlett 2006, 3540

[16] Jeyakumar, K.; Chand, D. K. Tetrahedron Lett. 2006, 47, 4573.

[17] (a) Fuchigami, T.; Inagi, S. Acc. Chem. Res. 2020, 53, 322.

(b) Nyffeler, P. T.; Duron, S. G.; Burkart, M. D.; Vincent, S. P.; Wong, C.-H. Angew. Chem., Int. Ed. 2005, 44, 192.

[18] Vincent, S. P.; Burkart, M. D.; Tsai, C.-Y.; Zhang, Z.; Wong, C.-H.
J. Org. Chem. 1999, 64, 5264.

[19] Kirihara, M.; Naito, S.; Ishizuka, Y.; Hanai, H.; Noguchi, T. Tetrahedron Lett. 2011, 52, 3086.

[20] Hu, J.; Yang, Y.; Lou, Z.; Ni, C.; Hu, J. Chin. J. Chem. 2018, 36, 1202.

[21] Bian, M.; Xu, F.; Ma, C. Synthesis 2007, 2951.

[22] Cheng, Z.; Sun, P.; Tang, A.; Jin, W.; Liu, C. Org. Lett. 2019, 21, 8925.

[23] Supale, A. R.; Gokavi, G. S. Phosphorus, Sulfur Silicon Relat. Elem. 2010, 185, 725 .

[24] Yang, C.; Jin, Q.; Zhang, H.; Liao, J.; Zhu, J.; Yu, B.; Deng, J. Green Chem. 2009, 11, 1401.

[25] Hanson, P.; Hendrickx, R. A. A. J.; Smith, J. R. L. Org. Biomol. Chem. 2008, 6, 745 .

(Zhao, C.) 